\title{
Exploring Determinants of M-Government Services: A Study from the Citizens' Persbective in Saudi Arabia
}

\author{
Mohammed Alonazi \\ University of Sussex \\ Informatics Department \\ Brighton, UK \\ Email: M.Alonazi@sussex.ac.uk
}

\author{
Natalia Beloff \\ University of Sussex \\ Informatics Department \\ Brighton, UK \\ Email: N.Beloff@sussex.ac.uk
}

\author{
Martin White \\ University of Sussex \\ Informatics Department \\ Brighton, UK \\ Email: M.White@sussex.ac.uk
}

\begin{abstract}
The government of Saudi Arabia has adopted MGovernment for the effective delivery of services. One advantage that it offers is unique opportunities for real-time and personalized access to government information and services. However, a low adoption rate of m-Government services by citizens is a common problem in Arab countries, including Saudi Arabia, despite the best efforts of the Saudi government. Therefore, this paper explores the determinants of citizens' intention to adopt and use m-Government services, in order to increase the adoption rate. This study was based on the Mobile Government Adoption and Utilization Model (MGAUM) that was developed for the purpose. Data was collected, and the final sample consisted of 1,286 valid responses. The descriptive analysis presented in this paper indicates that all the proposed factors in our MGAUM model were statistically significant in influencing citizens' intention to adopt and use m-Government services.
\end{abstract}

\section{INTRODUCTION}

G overnments from across the world have digitized their services to citizens through mobile technologies and the Internet, which has arguably improved communication between citizens and their governments, to provide better access to services and information, as well as improving government accountability, transparency and public governance [1], [2]. In this study, m-Government is defined as the use of mobile technology to deliver and improve eGovernment services and information to citizens, commercial organisations and all government agencies. Previous studies have either regarded $\mathrm{m}$-Government as separate to e-Government or as an extension or replacement of it [3], [4]. As it offers the public a valuable extra means to access services and information, it can be considered an advance in government service delivery [5]. Although eGovernment and m-Government work on the same principle, the latter is distinguished by features that are particular to it: Citizens can access the network from anywhere and at any time [6]; and can instantly receive messages from government service providers on their mobiles [7]. The mobile phone has recently become the primary way people communicate and has thus arguably become a part of everyday life for many people [8]. Therefore, being able to access government services via mobile devices might be the best route for citizens. Accessing government services and information on their phones means citizens neither have to visit the service provider in person nor go home to use their computer in order to do this [7], [9]. Access to the Internet may depend on economic factors, i.e. the extent of Internet access in a particular country, and how many citizens have access to computers providing mobile services can overcome these limitations [5].

An m-Government system provided by wireless technology, will give citizens opportunities for personalized access to government services and information in real time [10], [6]. This is especially beneficial for users in remote areas as m-Government services have the advantages of being affordable and easily and immediately accessible. Further, a relatively low level of digital literacy is required to operate them successfully [11], [6]. Given these features, the adoption of an m-Government system benefits both citizens and governments.

The Mobile Government Adoption and Utilization Model (MGAUM) has been developed as a framework from which to analyse factors affecting adoption and use of $\mathrm{m}$ Government services [12]. This study aims to investigate and understand Saudi citizens' perceptions towards the adoption and utilization of m-government services in developing countries, particularly Saudi Arabia, in order to increase the adoption rate of m-government services.

\section{THE RESEARCH METHODOLOGY}

The study was conducted in Saudi Arabia; and the questionnaire was distributed to adult Saudi citizens. The total valid responses constituted a sample of 1,286 Saudi citizens. SPSS was used to analyse the survey study data. The survey questionnaire was developed and modified from items used in previous research into both e-Government and m-Government. All items were measured with a 5-point Likert scale.

\section{A. Reliability and validity of the study}

To be considered reliable, a research instrument needs to produce similar results if used in comparable conditions and be relatively free of errors [13]. As part of the pilot study for 
this research, Cronbach's Alpha was chosen to assess the reliability of the questionnaire, and establish the internal consistency of the constructs used. Table 1 indicates the Cronbach's Alpha results for the complete research instrument, and demonstrates that the reliability of each of the constructs (independent and dependent variables) lies within the range of what is thought of as acceptable in academic research.

Face and content validity were chosen to be investigated rather than construct or criterion validity. The face validity of each item was ascertained in the pilot study to ensure that the model's factors measured what they were intended to measure; and items deemed to lack sufficient clarity, unambiguity or relevance were deleted or revised accordingly.

TABLE1: INTERNAL CONSISTENCY OF THE STUDY SURVEY INSTRUMENT

\begin{tabular}{|l|c|c|}
\hline \multicolumn{1}{|c|}{ Measured Variable } & No of Items & Cronbach's Alpha \\
\hline Perceived Usefulness (PU) & 7 & .898 \\
\hline Perceived Ease of Use (PEOU) & 4 & .862 \\
\hline Social Influence (SI) & 3 & .779 \\
\hline Perceived Compatibility (PCOM) & 2 & .868 \\
\hline Perceived Trust (PT) & 7 & .715 \\
\hline Culture (CULT) & 5 & .616 \\
\hline Awareness (AW) & 4 & .842 \\
\hline Perceived Mobility (PM) & 3 & .776 \\
\hline Citizens Service Quality (CSQ) & 8 & .920 \\
\hline System Quality (SQ) & 7 & .745 \\
\hline Intention to use (ITU) & 4 & .894 \\
\hline
\end{tabular}

\section{RESEARCH FINDINGS AND DISCUSSION}

This section provides an overview of respondents' demographic characteristics; and a descriptive analysis for each factor proposed in the MGAUM is given in order to explain their impact on citizens' intention to adopt and use $\mathrm{m}$-Government services in Saudi Arabia.

\section{A. Respondents' demographic data}

In the final sample 813 participants were male $(63.2 \%)$ and 473 were female $(36.8 \%)$. The highest percentage of participants was in the 18-30 age group, the largest number held a bachelor's degree and over half the participants (46.7\%) were government employees. All participants had Smartphone devices, with a large majority using mobiles and the Internet in daily life (96.4\% and $93.3 \%$ respectively). Approximately three-quarters of the respondents $(76.7 \%)$ have some knowledge about m-Government services in Saudi Arabia, whereas $22.9 \%$ had no knowledge. Moreover, the majority of participants $(90.5 \%)$ already used mGovernment services, but $9.5 \%$ had never used them. Participants who had already used m-Government services were asked to rate their general experience: $40.3 \%$ were very satisfied, $44.3 \%$ were satisfied to some extent, with only $5.9 \%$ not satisfied. Furthermore, $22.6 \%$ reported that the requirements of the intended $\mathrm{m}$-Government services were not clear and $26.6 \%$ reported that the system quality of $\mathrm{m}$ Government services was not good.

\section{B. The Measures of central tendency and Likert Scale}

A central tendency sums up an entire set of differing values, so the mean, median or mode is used according to what is most appropriate for the specific conditions being described. The mean is the most common measure of central tendency and was used in this study [14].

A Likert scale, was chosen as the main instrument in this study's questionnaire, as the simplest and most practical way to measure strength of opinion; and a review of the literature shows that is most commonly and successfully employed in IS research [14,15].

Weighted averages were calculated for the Likert scales, from Strongly Agree=1 to Strongly Disagree=5, (see Table 2) so that the tendency of the composite scores could be ascertained. The numbers entered into SPSS represent 'weight' and the weighted averages for the scale needs to be calculated to understand means. The results can be interpreted to show how influential (or not) each factor is [16].

TABLE 2: WEIGHTED AVERAGES FOR 5-POINT LIKERT SCALES

\begin{tabular}{|c|c|c|}
\hline Weighted average & Result & Result Interpretation \\
\hline $1-1.79$ & Strongly agree & Very influential \\
\hline $1.80-2.59$ & Agree & Influential \\
\hline $2.60-3.39$ & Neutral & Neutral or do not know \\
\hline $3.40-4.19$ & Disagree/ & Uninfluential \\
\hline $4.20-5$ & Strongly disagree & Very uninfluential \\
\hline
\end{tabular}

\section{Descriptive analysis of data}

The data collected was analysed with reference to each of the constructs in the MGAUM [12]. Participants' attitudes, intentions and behaviour towards adopting and using Saudi m-government services were explored by means of responses to statements for which Likert scores could be calculated.

TABLE 3: RESULT FOR ALL FACTORS

\begin{tabular}{|c|c|c|c|c|}
\hline Factor & Items & Mean & S.D. & Result interpretation \\
\hline PU & 7 & 1.3900 & .49876 & Very influential \\
\hline PEOU & 4 & 1.5733 & .61942 & Very influential \\
\hline CULT & 5 & 1.9997 & .69224 & Influential \\
\hline PT & 7 & 2.3586 & .62882 & Influential \\
\hline SI & 7 & 1.6516 & .62692 & Very influential \\
\hline PCOM & 2 & 1.5638 & .66208 & Very influential \\
\hline AW & 4 & 2.1763 & .87356 & Influential \\
\hline CSQ & 8 & 2.0852 & .69808 & Influential \\
\hline SQ & 7 & 1.8891 & .54653 & Influential \\
\hline PM & 3 & 1.5625 & .57052 & Very influential \\
\hline
\end{tabular}

Table 3 summarizes the results of the descriptive analysis with an interpretation of all results.

Perceived Usefulness (PU): It was important to determine what users' perceptions were about the usefulness of $\mathrm{m}$ - 
Government, the advantages that they would gain from using m-Government services in terms of saving time, effort and money and how this influenced their behavioural intention.

Most (93.9\%) agreed that using mobile government services would be useful. Participants believed that using them would enable government transactions to be effected more quickly as well as saving them time, money and effort $(94.4 \%)$ and enable them to perform transactions from any location (96\%); 89.6\% believed that using m-Government services would make communication between a government agency and citizens easier through text message, applications and e-mail; and over $94.2 \%$ agreed that using mobile government services would remind them of important dates in order to conduct or receive government transactions in sufficient time or at the right time. Using features on mobile devices that are not found on the website, such as reminders, location and camera were thought to add value to services.

The factor was interpreted as significantly influential on citizens' intention to adopt and use m-Government services; and that use of $\mathrm{m}$-Government services would increase when users perceive their benefits. This indicated that governments should take into consideration the requirements and needs of users to be met before implementing any services. The findings of this study are consistent with previous empirical investigations of factors that might affect the adoption of $\mathrm{m}$ Government services in Egypt [17], and Taiwan [18].

Perceived Ease of Use (PEOU): The vast majority of participants $(93.7 \%)$ agreed that learning to use mobile government services would be easy; and $92 \%$ believed that interactions with m-Government would be clear and understandable; $86.2 \%$ believed that using $\mathrm{m}$-Government services required little skill and effort.

The total score for the PEOU factor was 1.5733 (see Table3), which indicated that PEOU is very influential on users' intention to adopt and use M-Government services. The result suggests that the user experience is the first step in adoption; and if a user finds m-Government services easy to use and that it saves time and effort, this impacts positively on behavioural intention to adopt and use them. If services are easy to use, and people do not have to rely on asking for help from another person to use the application, the number of users will increase. Thus, PU and PEOU are essential factors in the MGAUM, and any theoretical framework which seeks to analyse intention, adoption and use of $\mathrm{m}$ government in the Saudi context or similar contexts in developing countries. The findings are with line with previous studies which found that PEOU is an important factor in determining intention to use [18], [19].

Culture (CULT): The concept of culture is complex and multi-dimensional, and contains many different aspects, for example, religion, social structure, language, political institutions, education and economics [20]. The behavioural intentions of Arab users are very much influenced by social values, interpersonal relationships and other issues related to religion [21]. CULT was measured by questions relating to central cultural aspects including Image, Resistance to Change and Interpersonal social networks (wasta or social connection and nepotism).

With respect to the influence of Image, participants were asked if they felt that using m-Government services would enhance their social status and make them feel more sophisticated; and (82.2\%) agreed that it would. Participants believed that using mobile government services would reduce the influence of interpersonal networks (wasta) $(85.2 \%)$ and prevent any negative influence on their transaction by uncooperative employees (87.9\%). Concerning Resistance to Change, only $27.2 \%$ of participants agreed that face-to-face dealings were better than using m-government, $17.4 \%$ of them were neutral, and $55.4 \%$ disagreed entirely. Furthermore, $21.8 \%$ of participants agreed that visiting agencies to track transactions was preferable to online tracking, $64.4 \%$ disagreed and $13.8 \%$ were neutral. The composite of the CULT factor was 1.9997, a result that indicated that CULT is influential on users' intention to adopt and use of m-Government services. This corresponds with findings by other studies in the literature, that revealed that social and cultural aspects were significant influences on Saudis' intention to adopt and use e-Government systems from both citizens' and employee's perspectives [22]; and that the main barriers to Omanis adopting e-Government were cultural rather than technical issues [23].

Perceived Trust (PT): Different aspects of PT such as risks to privacy (sharing and storing personal information), security and trust were measured. Participants were asked if they felt that the Internet was not safe to be used for dealing with the government; and $41.0 \%$ believed that the Internet was safe, while $33.3 \%$ believed it was safe, and $25.7 \%$ were neutral. This indicates some distrust in the Internet. By contrast, $73.9 \%$ of participants agreed that mobile government services were a safe and trustworthy environment in which to conduct government transactions, $20.8 \%$ were neutral and $5.3 \%$ disagreed. This indicates that citizens' trust of m-Government services is high, and this might well have a positive influence on citizens' intention to use m-Government services. However, regarding whether providing personal information was safe $42.6 \%$ agreed, $19.8 \%$ were neutral and $37.6 \%$ disagreed; and $33 \%$ agreed their data could be misused when stored by m-Government systems, $24.3 \%$ were neutral and $42.8 \%$ disagreed. The total score for PT was 2.3586, indicating that PT is influential on intention to use m-Government in line with numerous studies that have noted that perceived trust was a significant factor [18], [24].

Social Influence (SI): This factor addresses users' perception of the effect of social influence and how this would encourage intention to adopt and use m-Government services. A large majority (80.5\%) agreed that people important to them would think they should use mobile government services, $15.8 \%$ of them were neutral, and $3.7 \%$ 
disagreed. $89.3 \%$ of participants said they would be encouraged to use m-Government services by their families and friends. The vast majority of participants $(92.3 \%)$ intended to use m-Government services because it was the current trend. The composite of the SI factor was 1.6516, which indicates that SI is very influential on users' adoption and use; a result consistent with previous studies [24]. In a context like Saudi Arabia, where communities are very close, SI is a very important factor to include in the MGAUM, interestingly the desire to be seen by significant others as 'following the trend' was revealed as a powerful incentive in this context.

Perceived Compatibility (PCOM): This factor focuses on how users perceive the compatibility of m-Government services with their lifestyle and behaviour, and how this affects and encourages their intention to use m-Government services.

The vast majority $(91.4 \%)$ of participants believed that using m-Government services would fit well with their lifestyles as well as being the way they liked to conduct government transactions $(89.7 \%)$. The total score for PCOM was 1.5708 which indicates that this factor is very influential on intention to use; and that a high level of compatibility with the innovation would increase users' intention to adopt and use it. This finding is in line with other studies [24], [17]. Unlike previous research, this study included a high number of Saudi female participants. In a society where contact between sexes is sensitive for religious and cultural reasons, conducting government transactions on their phones arguably gives Saudi women both privacy and removes the need for face-to-face interactions with male government officials.

Awareness $(\boldsymbol{A W})$ : is the first stage where users experience a new service offered by the government. Participants were asked about which advertising methods could affect citizens' awareness of m-Government services and encourage their intention to adopt and use.

Many participants (76.7\%) believed that they had a good level of knowledge about the benefits, features and services of m-Government, $14.9 \%$ were neutral, and $8.4 \%$ did not. Almost $68.6 \%$ of participants agreed it was easy to find out if a government agency offered its services via mobile devices, $17 \%$ of participants were neutral and $14.4 \%$ disagreed. Furthermore, $68.8 \%$ of participants agreed they had received enough information and guidance on how to use mobile government services, $14.9 \%$ disagreed and $16.3 \%$ were neutral. Also $66.6 \%$ were satisfied with the current awareness campaigns and advertising about m-Government services in Saudi Arabia, 16.4\% were neutral and 17.0\% were not satisfied. It is thus likely that AW positively influences citizens' intention to adopt and use mGovernment services, in line with other findings [17], [25].

Citizen Service Quality (CSQ): Three main service quality dimensions were used to measure service quality in this study, i.e. reliability, responsiveness and empathy; and designed to measure customers' evaluation of the overall experience of services and explaining the difference between users' perceptions and their expectations of the services offered by the government.

Participants were asked to what extent that they believed that $\mathrm{m}$-Government service providers give 'a prompt service with a good response'; $75.6 \%$ agreed they did, $19.2 \%$ were neutral, and $5.2 \%$ disagreed. Similarly, most participants believed that $\mathrm{m}$-Government service providers offered helpful assistance through SMS.

When asked whether they believed that information provided through m-Government services was accurate. $76.4 \%$ agreed it was, $19.9 \%$ were neutral and $3.7 \%$ disagreed. Furthermore, $69.4 \%$ of participants believed that $\mathrm{m}$-Government service providers showed a sincere interest in solving citizens' problems, $24.8 \%$ were neutral, and $5.8 \%$ disagreed. The result echoes findings in Jordan [26] and Saudi Arabia [27] that the service quality dimension impacts significantly on citizen satisfaction and behavioural intention.

System Quality (SQ): This includes the technical aspects that are recognised by users, and which can affect their willingness and intention to adopt and use m-Government services. Regarding whether the speed of launching mGovernment services applications or websites would affect participants' intention to use it, $79.1 \%$ agreed it would, $13.5 \%$ were neutral and $7.5 \%$ disagreed.

$81.6 \%$ believed that $\mathrm{m}$-Government was easy to navigate and that it provided good navigation functions; in contrast, $14.2 \%$ were neutral and only $4.2 \%$ disagreed. In respect of the existence of technical errors, such as applications crashing, links not working and unresponsiveness, (application/website), as well as the bad layout and unattractive interfaces of m-Government services $79.7 \%$ and $73.3 \%$ of participants respectively, agreed that these elements would reduce their willingness to use it. $86.2 \%$ of participants agreed that services should be compatible with mobile devices such as GPS and camera. Furthermore, $80.2 \%$ and $76.8 \%$ respectively believed that m-Government services provided fast responses to their enquiries as well as up-to-date information. The composite of the SQ factor was 1.8897, which indicated that SQ is influential on users' intention to adopt and use m-Government services. The results echo studies that indicated that when the system quality of m-Government services increases, citizens' intention to adopt and use them will also increase [26], [27].

Perceived Mobility (PM): The majority (89.8\%) of participants expected to be able to use m-Government services anywhere and at any time; and found mobile government services were easily accessible, portable and easy-to-use on different models of Smartphone. 94.6\% agreed it was important to get critical alert notifications on their mobiles from government agencies via text or email regarding passport renewal, traffic penalties and emergency cases whilst they were on the move. The composite of the PM factor was 1.5625 which indicates that PM is very influential on the intention to adopt and use m-Government services, and that citizens in Saudi Arabia value the ability to constantly access government services and information from 
any location. The findings for this factor are consistent with previous studies in China [28].

The statistical analysis and discussion above allow us to estimate the most influential factors for increasing the intention to use m-Government services in Saudi Arabia.

\section{CONCLUSION}

The researchers constructed the MGAUM model to identify factors revealed by the literature and personal professional experience to be likely to influence Saudi citizens' intention to adopt and use m-Government services.

The result of descriptive analysis presented in this paper indicates that all the proposed factors in MGAUM model (PU, PEOU, CULT, SI, PCOM, PT, AW, CSQ, SQ and PM) were statistically significant factors in influencing Saudi citizens' intention to adopt and use m-Government services and when properly addressed could increase the adoption rate of $\mathrm{m}$-Government services.

We intend the results to provide a valuable insight into the main factors that influence citizen intention to adopt and use m-Government services in Saudi Arabia; which will be useful for researchers, the ICT industry and for policymakers who are keen to find strategies that result in quicker and more efficient take-up of such services.

This study added several contributions to theory and practice in the field of $\mathrm{m}$-Government adoption and use. Firstly, this research developed the Mobile Government Adoption and Utilization Model (MGAUM) to analyse factors that affect users' adoption and use of m-government. MGAUM integrates the Technology Acceptance Model with a number of social, cultural and technological factors, taken from other recognized theoretical acceptance models that have been identified as key factors in the literature. Secondly, the MGAUM is empirically tested and validated by collecting and analysing primary data from the citizens' perspectives. Thirdly, this is one of the first few studies investigating the adoption and utilization of $\mathrm{m}$-government in Saudi Arabia.

\section{REFERENCES}

[1] S. Alghamdi, N. Beloff, Towards a comprehensive model for eGovernment adoption and utilisation analysis: The case of Saudi Arabia, 2014 Fed. Conf. Comput. Sci. Inf. Syst. FedCSIS 2014. 2 (2014) 1217-1225. doi:10.15439/2014F146.

[2] O. Al-Hujran, M.M. Al-Debei, A. Chatfield, M. Migdadi, The imperative of influencing citizen attitude toward e-government adoption and use, Comput. Human Behav. 53 (2015) 189-203. doi:10.1016/j.chb.2015.06.025.

[3] H.J. Scholl, The mobility paradigm in electronic government theory and practice: A strategic framework, in: Euro Mob. Gov. (Euro MGov) Conf., 2005: pp. 1-10.

[4] S. Alotaibi, D. Roussinov, Developing and Validating an Instrument for Measuring Mobile Government Adoption in Saudi Arabia, World Acad. Sci. Eng. Technol. Int. J. Soc. Behav. Educ. Econ. Bus. Ind. Eng. 10 (2016) 710-716.

[5] I. Kushchu, M.H. Kuscu, From E-government to M-government: Facing the Inevitable, Proc. 3rd Eur. Conf. EGovernment. (2003) 253-260. http://citeseerx.ist.psu.edu.

[6] M. Ntaliani, C. Costopoulou, S. Karetsos, Mobile government: A challenge for agriculture, Gov. Inf. Q. 25 (2008) 699-716. doi:10.1016/j.giq.2007.04.010.
[7] I. Almarashdeh, M.K. Alsmadi, How to make them use it? Citizens acceptance of M-government, Appl. Comput. Informatics. 13 (2017) 1-6. doi:10.1016/j.aci.2017.04.001.

[8] L.C. Serra, L.P. Carvalho, L.P. Ferreira, J.B.S. Vaz, A.P. Freire, Accessibility Evaluation of E-Government Mobile Applications in Brazil, Procedia Comput. Sci. 67 (2015) 348-357. doi:10.1016/j.procs.2015.09.279.

[9] R. Lallana, E-government for development m-government: Mobile/wireless applications, in: 2004

[10] A.M. Alsenaidy, T. Ahmad, A Review of Current State M Government in Saudi, Glob. Eng. Technol. Rev. (2012) 5-8.

[11] Y. Liu, H. Li, V. Kostakos, J. Goncalves, S. Hosio, F. Hu, An empirical investigation of mobile government adoption in rural China: A case study in Zhejiang province, Gov. Inf. Q. 31 (2014) 432-442. doi:10.1016/j.giq.2014.02.008.

[12] M. Alonazi, N. Beloff, M. White, MGAUM - Towards a Mobile Government Adoption and Utilization Model: The Case of Saudi Arabia, Int. J. Business, Hum. Soc. Sci. 12 (2018) 459-466.

[13] N. Blunch, Introduction to Structural Equation Modelling Using IBM SPSS Statistics and AMOS", Second Edition, SAGE publication Ltd, 2013.

[14] Viswanathan, Madhubalan, S. Sudman, M. Johnson, Maximum versus meaningful discrimination in scale response: Implications for validity of measurement of consumer perceptions about products, J. Bus. Res. 75 (2004) 108-124. doi:10.1016/S0148-2963(01)00296-X.

[15] V. Venkatesh, M.G. Morris, G.B. Davis, F.D. Davis, User Acceptance of Information Technology: Toward a Unified View, MIS Q. 27 (2003) 425-478. doi:10.1017/CBO9781107415324.004.

[16] W.A. Alfarra, Analysing Questionnaires Data Using SPSS. Programs and Foreign Affairs Department, World Assembly of Muslim Youth., [Arabic Source]. https://www.kantakji.com/media/9166/edu.pdf.

[17] H. Abdelghaffar, Y. Magdy, The Adoption of Mobile Government Services in Developing Countries: The Case of Egypt, Int. J. Inf. Commun. Technol. Res. 2 (2012) 333-341.

[18] S.Y. Hung, C.M. Chang, S.R. Kuo, User acceptance of mobile egovernment services: An empirical study, Gov. Inf. Q. 30 (2013) 3344. doi:10.1016/j.giq.2012.07.008.

[19] S. Alghamdi, N. Beloff, Exploring Determinants of Adoption and Higher Utilisation for E-Government: A Study from Business Sector Perspective in Saudi Arabia, 5 (2015) 1469-1479. doi:10.15439/2015F257.

[20] L. Chang, Cross-Cultural Differences in International Management Using Kluckhohn-Strodtbeck Framework, J. Am. Acad. Bus. 2 (2002) 20-27.

[21] P.J.-H. Hu, S.S. Al-Gahtani, H.-F. Hu, Arabian workers' acceptance of computer technology: A model comparison perspective, J. Glob. Inf. Manag. (2014). doi:10.4018/jgim.2014040101.

[22] R. HMBP, Role of National Culture on the Use of e-Government Services in Sri Lanka, J. Bus. Financ. Aff. 5 (2016). doi:10.4172/2167-0234.1000182.

[23] S.J. Naqvi, H. Al-shihi, M-Government Services Initiatives in Oman, 6 (2009).

[24] C. Sellitto, M.W.L. Fong, An investigation of mobile payment (mpayment) services in Thailand, Asia-Pacific J. Bus. Adm. 8 (2015) 37-54. doi:10.1108/APJBA-10-2014-0119.

[25] S.A. Al-Somali, R. Gholami, B. Clegg, An investigation into the acceptance of online banking in Saudi Arabia, Technovation. 29 (2009) 130-141. doi:10.1016/j.technovation.2008.07.004.

[26] F.T. Qutaishat, Users' Perceptions towards Website Quality and Its Effect on Intention to Use E-government Services in Jordan, Int. Bus. Res. 6 (2012) 97-105. doi:10.5539/ibr.v6n1p97.

[27] A.M. Baabdullah, A.A. Alalwan, N.P. Rana, H. Kizgin, P. Patil, Consumer use of mobile banking (M-Banking) in Saudi Arabia: Towards an integrated model, Int. J. Inf. Manage. 44 (2019) 38-52. doi:10.1016/j.ijinfomgt.2018.09.002.

[28] Y.S. Yen, F.S. Wu, Predicting the adoption of mobile financial services: The impacts of perceived mobility and personal habit, Comput. HumanB ehav. 65 (2016) 31-42. doi:10.1016/j.chb.2016.08.017. 